\section{$\underset{\substack{\text { hommes } \\ \text { \& migrations }}}{ }$}

\section{Hommes \& migrations}

Revue française de référence sur les dynamiques

migratoires

$1312 \mid 2015$

Diasporas iraniennes

\title{
Frédéric Boyer, Quelle terreur en nous ne veut pas finir ?
}

P.O.L., 2015, 103 pages, $9 €$

\section{Mustapha Harzoune}

\section{(2) OpenEdition Journals}

Édition électronique

URL : http://journals.openedition.org/hommesmigrations/3541

DOI : 10.4000/hommesmigrations.3541

ISSN : 2262-3353

Éditeur

Musée national de l'histoire de l'immigration

Édition imprimée

Date de publication : 1 octobre 2015

Pagination : 158-159

ISBN : 978-2-919040-33-9

ISSN : 1142-852X

Référence électronique

Mustapha Harzoune, «Frédéric Boyer, Quelle terreur en nous ne veut pas finir? », Hommes \& migrations

[En ligne], 1312 | 2015, mis en ligne le 31 mai 2016, consulté le 23 septembre 2020. URL : http://

journals.openedition.org/hommesmigrations/3541 ; DOI : https://doi.org/10.4000/

hommesmigrations.3541 


\section{LIVRES}

\section{Frédéric Boyer Quelle terreur en nous ne veut pas finir? \\ P.O.L., 2015,103 pages, $9 €$}

Quelle terreur en nous ne veut pas finir ? a été publié après les attentats de janvier, avant que la photo du petit Aylan ne fasse le tour de la planète, avant l'arrivée de réfugiés et l'érection sur le sol européen de barrières de barbelés et de murs, avant les attentats du 13 novembre. Et depuis, de drame en drame, de tragédie en tragédie, d'urgence en urgence, la lecture de ce "petit livre", comme dit son auteur, devient, jour après jour, cruciale, vitale. Car il s'agit de contenir cette terreur en nous qui, sondage après sondage, semble se répandre et avec elle ces idées de fermeture et de protection, de forteresse à défendre, de passé à protéger, d'identité menacée et malheureuse, de mémoire figée; la crainte du grand remplacement. Une terreur contagieuse, qui se nourrit de fantasmes, de peurs irraisonnées, disproportionnées. Une terreur qui conduit à la résurgence des idéologies de division, de classement, aux "relents tristes de la vieille souveraineté" qui s'autorise le droit de vie et de mort, le bannissement, la production de boucs émissaires et le retour de la race. Le tout emballé dans une pseudo-morale - car il en faut une imprégnée de certitudes républicaines ou civilisationnelles, du “tout n'est pas possible" ou pire du "mieux faut rester chez vous, mieux faut rester vousmêmes et vous nous remercierez".

Cette terreur repose sur une illusion : I'illusion de la permanence, de l'immortalité des civilisations, des cultures et peut-être des hommes. Croire à l'imprescriptibilité des idéologies, des religions, des romans nationaux. II suffirait de fermer la porte, de rester entre soi pour se protéger, se pérenniser, quand l'histoire enseigne le contraire : il n'y a de pérennité que dans le mouvement, le changement, la plasticité, la réinvention, l'échange, la "bâtardise"(Amin Maalouf), dans le questionnement de l'accueil et de sa nécessité, dans l'hospitalité et la présence de l'autre, dans I'horizon et l'espoir d'une autre histoire. "Est-ce que nous ne serions pas plus forts en nous agrandissant?" demande Frédéric Boyer ,qui rappelle L'Art français de la guerre d'Alexis Jenni.

Il faut risquer notre intégrité, notre histoire commune, "sous peine de ne pas préserver notre propre humanité". Aujourd'hui, plus encore qu'hier, il faut "accepter d'être liés par obligation de tous envers tous" (Dostoïevski). L'exigence éthique rejoint l'effectivité d'un monde devenu, par quelque bout qu'on le prenne, interdépendant.

C'est de morale dont Frédéric Boyer nous entretient. La vraie, celle qui "ne tranche pas", qui "répare", celle qui tente de renouer les fils. À ceux qui l'accusent, lui et ses semblables, de "bons sentiments", d'"angélique bêtise", il répond que "ce qui demande le plus de courage" est justement de "ne pas rester entre soi". Comme Pennac, Laabi ou Belaskri', avec saint Augustin et son 
"amour de l'amour" - "Peut-on aimer son frère sans aimer l'amour ?" - il assume crânement ses engagements moraux, et cela est tout sauf facile ! Tout sauf angélique! Rappelant le Jean Amrouche des Chants berbères de Kabylie (1938), Frédéric Boyer écrit: "Je prends le parti de l'innocence, justement, humblement, je prends le parti de ne rien comprendre, de ne rien savoir, et qui est souvent la seule force de l'enfance, qui est souvent la seule ressource nous menant à l'innocence, la seule voie

\section{Boualem Sansal 2084. \\ La fin du monde \\ Gallimard, 2015, 274 pages, $19,50 €$}

C'est un grand classique des critiques et des "professionnels de la parole" que de tirer la couverture à soi, de se donner le beau rôle et bonne conscience (historique, civilisationnel, etc.), de s'affranchir et de gommer les subtilités d'une œuvre pour y aller franco en criant haro sur l'Autre et, en l'occurrence, son islam de dégénérés. Cela fait du bien, rassure, et tout n'est pas à jeter. 2084 est pour le coup un bel os. Pour autant, il ne faudrait pas s'arrêter là et réduire l'œuvre à sa réception, et l'écrivain à un rôle de "bon client" des plateaux et des gazettes.

Certes, il est difficile de ne pas lire dans 2084 une description (et non une prévision et encore moins une prophétie) de l'emprise et des mécanismes tota- éthique. (...) Savoir d'instinct, savoir sans le comprendre que la seule force, la seule valeur, la seule dignité, c'est de ne pas comprendre si comprendre nous fait renoncer à l'amour de l'autre. Voilà ce qui fonde, voilà ce qui fait la légitimité d'une existence."

Mustapha Harzoune

1. Voir Eux, c'est nous, L'instinct, le coeur et la raison de Daniel Pennac, Les éditeurs jeunesse avec les réfugiés, 2015 ; le poème J'atteste de Abdellatif Laâbi ou Yahia Belaskri, Les Fils du jour, Vents d'ailleurs, 2014.

litaires déjà à l'œuvre ici et là. Boualem Sansal est de ce peuple algérien meurtri par la terrible décennie 1990 et averti de ce qui en a constitué les prémices. Esprit libre et indépendant, il ne confond pas critique de la religion avec ce que d'autres (perroquets) réduisent et dénoncent comme étant de l'islamophobie. II ne faudrait pas, a contrario, renvoyer l'écrivain à ses commentateurs trop heureux d'agiter leur hochet civilisationnel, d'autant plus bruyant qu'il est amnésique.

Pour autant, 2084 traite bien d'un totalitarisme orwelien version islamiste. Nous sommes dans un empire encore imaginaire, l'Abistan, dominé par le Grand Commandeur entouré des 40 Honorables de la Juste Fraternité et des agents de l'Appareil capables d'épier les moindres pensées en s'insinuant dans les crânes "grâce à un appareil télépathique". Un seul livre renferme le passé et le futur et édicte les règles éternelles, le saint Gkabul, dans lequel Abi a consigné ses divins enseignements. Pour les ouailles abistanes, "Yölah est grand et 